\title{
Publisher Correction: Idiosyncratic choice bias naturally emerges from intrinsic stochasticity in neuronal dynamics
}

Lior Lebovich (D), Ran Darshan, Yoni Lavi(D), David Hansel and Yonatan Loewenstein (D)

Correction to: Nature Human Behaviour https://doi.org/10.1038/s41562-019-0682-7, published online 2 September 2019.

In the version of this article initially published online, reference \#4 should have been to:

"Linares, D., Aguilar-Lleyda, D. \& López-Moliner, J. Decoupling sensory from decisional choice biases in perceptual decision making. eLife 8, https://doi.org/10.7554/eLife.43994 (2019).” Additionally, the $y$-axis label in Fig. 2d should have read "Expected $p_{c w}$ ”.

The error has been corrected in the print, PDF and HTML versions of this article.

Original

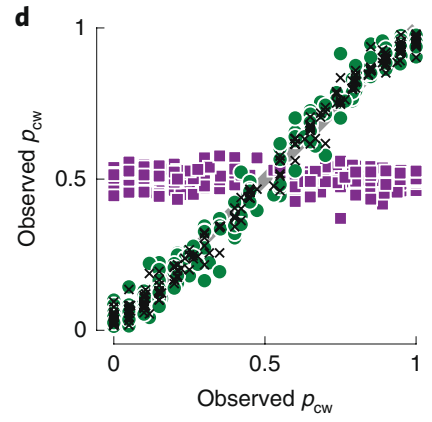

Corrected

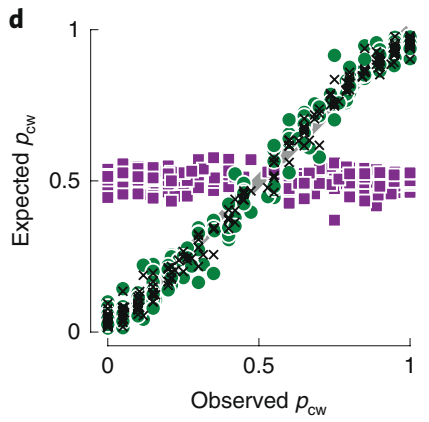

Fig. 2 | Original and Corrected.

Published online: 20 November 2019

https://doi.org/10.1038/s41562-019-0784-2

๑ The Author(s), under exclusive licence to Springer Nature Limited 2019 